\title{
A Optimum Resource Allot for Data in Code Division multiple access Contrary Link
}

\author{
${ }^{1}$ Mr.Nitish Meena, Dr. Nilesh parihar ${ }^{2}$ \\ ${ }^{I}$ Dept.of ECE, pratap University (MPGI) Jaipur, Rajasthan, India Dept. Of E.C.E Phd (pur.) \\ ${ }^{2}$ Dept.of ECE, Bhartiya Institute of Engineering \& Technology Sikar, Rajasthan ,India
}

\begin{abstract}
The optimum resource apportionment standpoint is studied for non-real-time users CDMA (code division multiple access) overturn link. A resource apportionment standpoint of concern includes channel coding, out strectching gain control and power allocation under the conventional receive operation. The restriction in the optimization include peak transmit power of the mobile station, total received power at the base station and QoS in the form of minimum SINR (Signal-to- Interference-plus- Noise-Ratio) for each user. The coding and flattening gain control can be share from the power apportionment strategy. Our results show that the optimal power allotment standpoint depends on the purpose function: avaricious standpoint is optimal to maximize the sum of throughput from each user, whereas a fair standpoint is optimal to maximize the product of throughput from each user. A unified approach is taken to derive the optimal policies, and it can also be applied to other power apportionment problems in CDMA (code division multiple access) reverse link. Numerical results of the channel volume which can be held are presented for both objectives along with the effect of QoS constraints Code division multi access, information rates land(arrive on the ground, alight) mobile radio cellular systems, nonlinear programming resource (source of wealth) management.
\end{abstract}

\section{Introduction}

A Direct Sequence-Code Division Multiple Access (DSCDMA) has been adopted as the core technology for multiple 3G mobile cellular systems including W-CDMA and CDMA2000. These3G systems provide a wide variety of different communication services, such as real-time services.

A including voice, video, and non-real-time or delay-tolerant data services including web browsing, $\mathrm{ftp}$, e-mail, etc. Since the multiple users interfere with each other in code division multiple access systems.

It is decisive to set aside and control the power and rate of each user. For those using the non-real-time services, their delay endurance can be taken advantage of to enhance system performance.

In this paper, we consider the optimum resource apportionment standpoint or the non-real-time users in DS-CDMA reverse link. It includes the apportionment of the users transmit power, the apportionment of the users' rate through the expansion gain control and the selection of the channel coding for error act of detecting or error correction. The objective is to optimize system performance.

A basis that each user's Quality-of Service (QoS) necessity is satisfied and the total interference to the realtime users in the same sector.The resource apportionment in CDMA systems has captured important compensation during the past years. provided a survey (act of measuring and recording characteristics of a plot of land) in on the characterization of the capacity region, i.e the capacity vector consisting of the able to be accomplised throughput for each user, and the corresponding power control .In our work, we are more curious in optimizing (improvenment of efficiency) the performance of the entire cell/sector as a replacement of that of each individual user. One important purpose in optimizing (improvenment of efficiency) system performance is to maximize the total sector/cell throughput or the ghostly efficiency. In the information abstract bounds are maximized. In the hypothetical optimal power allocation is obtained when the number of users access infinity. A common feature in the earlier work on this issue, comprising and is that the advanced multi-user demodulation is assume at the receiver. Since the advanced multi-user demodulation has not been involve in any of the current and proposed 3G DS-CDMA systems due to its calculative complexity, we do not assume it in our work.

Similar to this work, the throughput largest possible magnification based on the common matched-filter receiver is studied in throughput largest possible magnification but its devising is limiting since only the expansion gain control is measured. A part from the total throughput, in a more general form, optimum (maximum) resource allocation should be designed to maximize (magnify as much as possible) the sum of usefulness functions from each user. usefulness functions are formally defined in microeconomics and can be instinctively understood as a quantitative description of the users' satisfaction. As much as possible of the total throughput is one special case of this general formulation where the usefulness function is simply the through put. Although there has been no majority on the exact form of the user's usefulness as a function of its throughput, it is greatly recognized that for a hindrance tolerant data user, or an "elastic user" according to mischief in, such a function should be increasing, curving inward and continuous differ. Kelly proposed using 
the logarithm as the usefulness function in , and it leads to the "proportional fair" rate allocation standpoint in the wired network. Since then, this function and the proportional fair design have been studied in various problems in the wired networks. In the overall situation of wireless networks, the proportional fair principle has been adopted in the forward link scheduler design in the CDMA2000 1xEV-DO (1x progression Data Optimized) high speed packet data system . A similar idea has been applied to the CDMA2000 reverse link in but it requires the multiple users be Time-Division-Multiplexed (TDM) rather than Code-Division- Multiplexed (CDM). Thus significant signaling.

A general expenses must be added and backward suitability can be lost. One of the results in this paper shows the proportional fair allotment for the reverse link on the CDMA basis. In this paper, we concieve the optimal resource allotment as usefullness maximization problems. The constraints in our optimization (improvement of efficeiency) includes peak transmit power, total received power from all the data users and a minimum Signal-to- Interference-plus-Noise-Ratio (SINR) for each user for QoS or fairness obligation. In our work, a general methodology in searching and proving the optimum policy is discovered, which is applicable to a broad range of resource allocation designs.

As examples, we show the optimal policies for two types of usefulness functions: one is linear with respect to the throughput which makes the optimization the same as the largest possible magnification of the total throughput the other is the logarithm of the throughput, which makes the optimization (improvement of efficiency) equivalent to the largest possible magnification of the product of users' yield. The product maximization leads to a policy similar to the "proportional fair" apportionment but the presence of the advanced multi-user scrambling of wave or signal make complex the policy and its derivation. For each of these two objectives, we consider each two formulations: one is with fixed coding but the distribution gain and power are controlled; the other is with optimal codes in the sense that the Shannon capacity for each single user is attained. In Code division multiple access systems, multiple users interfere with each other . The concavity in the something useful function only exists with respect to an individual user's power but not the power vector. This makes the non-linear improvement of efficiency much more stimulating However, our general method provides important structures which often result in simple search for the optimum (maximum) policies. The hypothesis of peak power constraint separates our work from previous works like where the constraint sare on the average transmit power. Moreover, the absence of the optimize advanced multi-user detection is another dividing line between our work and previous works. The rest of the paper is organized as follows. The improvement of competence problems with constraints are formulated in Section II. The methodology to solve the optimization problems is presented in Section III. In Section IV, the methodology (using scientific research method) presented in Section III is used to solve the problems defined in Section II. Numerical examples are presented in Section V.

\section{Optimization Technique}

We consider a time-slotted system. Let $M$ be the number of non-real-time data users in the CDMA (code division multipal access) cells and their reverse link channel gains are ordered as g1 > g2 > . > gM. Without losing generality, we assume that no two users have the same channel gain. Vector $\mathbf{g}=(\mathrm{g} 1, \mathrm{~g} 2, \cdots$, $\mathrm{gM}$ ) includes channel gains of all the users. We also assume that $\mathbf{g}$ is known to the base station at the start of each time plot and all gi, $\mathrm{i}=1,2, \cdots, \mathrm{M}$, remain constant during each time slot. Vector $\mathbf{p}=(\mathrm{p} 1, \mathrm{p} 2, \cdots, \mathrm{Pm})$ is the transmit power of all the users, allocated by the base station. We use vector $\mathbf{p}-\mathrm{i}=(\mathrm{p} 1, \mathrm{p} 2, \cdots, \mathrm{pi}-1$, $\mathrm{pi}+1, \cdots, \mathrm{pM})$ for the power of all the other users except user i. Suppose that the matched filter receiver is used at the base station, namely there is no advanced multiuser detection. Then, the Ratio (SINR) of user $i$, $\gamma \mathrm{i}$ becomes

$$
\gamma_{i}=\frac{p_{i} g_{i}}{I+\alpha \sum_{k \neq i} p_{k} g_{k}} \quad \text { where } \quad i=1,2, \cdots, M \text {. }
$$

In (1), parameter $\alpha$ is included for generality. According to [2], in practical systems, $\alpha \approx 1$.

As previously stated, we have two types of assumptions on the channel coding in this paper: one assumes an arbitrary but fixed coding at the symbol level; the other uses the Shannon coding, namely the 'optimum' codes achieving the information theoretical channel capacity for each individual user. In the first case (fixed coding), as shown in [7], the optimal spreading gain can be obtained separately from the power allocation. In the second case (Shannon coding), the codes can be determined by well-known result in information theory [16], again separately from the power allocation. Henceforth we focus on the power allocation part in this sheet. In the following, we list four example formula: two of them with fixed (stay) coding and the other than with Shannon coding; at the same time, two of them with sum of throughput and the other two with product of throughput (sum of log throughput). All of them are solved later in Section IV by our unified method presented in Section III. 


\section{A.Total Maximization With Fixed Coding}

Suppose we have chosen a specific but fixed coding scheme on the symbol level.We haves preading gain and power at our control. This problem is formulated in [7]. As shown there, the optimum spreading gain can be determined self-sufficiency from power allocation: at any power level, the optimal spreading gain is always inversely proportional to the chip-level SINR, where the proportion is dependent on the selected coding scheme. Consequently, maximizing the total through put is equivalent to maximizing the sum of SINR. Therefore, essentially we have the following optimization problem.

$$
\text { (P1) } \max _{\mathbf{p} \in \mathcal{C}} J_{1}\left(p_{1} g_{1}, p_{2} g_{2}, \cdots, p_{M} g_{M}\right)
$$

where

$$
J_{1}=\sum_{i=1}^{M} \frac{p_{i} g_{i}}{I+\alpha \sum_{k=1, k \neq i}^{M} p_{k} g_{k}} .
$$

Note that the code-dependent scaling factor and the total bandwidth term are suppressed in (2).

The constraint, $\mathrm{C}$, considered throughout the paper consists of three individual constraints by $\quad \mathrm{C}=\mathrm{CP}_{\max } \cap \mathrm{C}$ $\mathrm{QoS} \cap \mathrm{CP}_{\max } \mathrm{R}$

Each constraint is defined as follow:

- $\mathrm{CP}_{\max }=\left\{\mathbf{p}: 0 \leq \mathrm{pi} \leq \mathrm{P}_{\max }\right.$, for all $\left.\mathrm{i}=1,2, \cdots, \mathrm{M}\right\}$, and it is the peak power constraint. This is due to the mobile stations' battery limitation and radiation regulation.

- $\mathrm{C}$ QoS $=\{\mathbf{p}: \gamma \mathrm{i} \geq \Lambda$, for all $\mathrm{i}=1,2, \cdots, \mathrm{M}\}$, and it is the QoS constraint. This QoS constraint is motivated by the observation that the optimal power allocation, without such a constraint, may be greedy and unfair.

- $\mathrm{CPmax} R=\left\{\mathbf{p}: \sum_{-} \mathrm{M}\right.$ i=1 Pi gi $\left.\leq \mathrm{P}_{\max } \mathrm{R}_{-}\right\}$, and it is the total received power constraint. It is due to the fact that the total received power from the data users is an interference.

to other classes of users, like the voice users in the same sector. We assume that the QoS constraint can be met for all the users. In other words, we assume that the feasibility condition is satisfied, and in practice, this can be achieved by network planning and admission control [19]. (P1) without QoS constraint is solved in [7] using elementary proof. Here we apply our general approach which greatly simplifies the derivation and extends their result.

\section{B. Maximizing firm Capacity}

In the above subsection, a specific coding is chosen and fixed. Suppose we relax this limitation and assume that the coding scheme on the symbol level is chosen to achieve the channel capacity for each single user without the multiuser detection. Based on the well known Shannon capacity result [16], we have the following optimization problem:

$$
(P 2) \max _{\mathbf{p} \in \mathcal{C}} J_{2}\left(p_{1} g_{1}, p_{2} g_{2}, \cdots, p_{M} g_{M}\right)
$$

where

$$
J_{2}=\sum_{i=1}^{M} \log \left(1+\frac{p_{i} g_{i}}{I+\alpha \sum_{k=1, k \neq i}^{M} p_{k} g_{k}}\right)
$$

The constraints are the same as the one in the previous formulation. All through this paper, for the convenience of notation, the bandwidth term is suppressed from the capacity formula and natural logarithm is used instead of ' $\log 2$ '. Note that the effect of spreading gain control is absorbed by the capacity-achieving codes. (P2) with $\alpha=1$ has been solved in [15] using main component proof. Here we apply our general approach which greatly simplifies the derivation and extends their result.

\section{Maximizing the Product of yield With firm Coding}

Since maximizing the total summed throughput even with QoS constraint may lead to unfair policy, it is proposed to consider maximizing the product of each user's throughput. This can be seen as a "proportional 
fair" formulation. If we assume a fixed coding scheme, the result on the optimal spreading gain in [7] still applies. Thus maximizing the product of throughput is equivalent to maximizing the product of SINR. Therefore we have the following optimization problem:

$$
\begin{gathered}
\text { (P3) } \max _{\mathbf{p} \in \mathcal{C}} J_{3}\left(p_{1} g_{1}, p_{2} g_{2}, \cdots, p_{M} g_{M}\right) \\
J_{3}=\prod_{i=1}^{M} \frac{p_{i} g_{i}}{I+\alpha \sum_{k=1, k \neq i}^{M} p_{k} g_{k}} .
\end{gathered}
$$

As seen later, even without the QoS constraint, the QoS is assured for each user by the optimal policy.

\section{Maximizing the Product of Channel Capacity}

We can have a similar devising based on the optimal coding at symbol level for each individual user without the multiuser detection. Therefore the optimization problem is as follows:

$$
\text { (P4) } \max _{\mathbf{p} \in \mathcal{C}} J_{4}\left(p_{1} g_{1}, p_{2} g_{2}, \cdots, p_{M} g_{M}\right)
$$

where

$$
J_{4}=\prod_{i=1}^{M} \log \left(1+\frac{p_{i} g_{i}}{I+\alpha \sum_{k=1, k \neq i}^{M} p_{k} g_{k}}\right) .
$$

\section{A. General Properties of Optimum Solutions} powers.

Define $\mathrm{xi}=$ pigi as the received power of user $\mathrm{i}$, and $\mathbf{x}=(\mathrm{x} 1, \mathrm{x} 2, \cdots \mathrm{xM})$ as the vector of received

In the sequel, we interchangeably use the received power vector $\mathbf{x}$ and the transmit power vector $\mathbf{p}$. The solution of the optimization problem begins with an application of the Kuhn-Tucker condition [17]. Note that each $\operatorname{Jn}(\mathbf{x})$ of the for devising, is symmetric to any interchange of user indices. This is generally the case in the reverse link resource allocation problems without the advanced multi-user detection. Few properties of $\mathbf{J}$ are drawn using this symmetry.

The following is a re-write of the optimization problem 1 with the restriction in a linear form:

$\operatorname{Max} \mathbf{x} \in \mathrm{C} \mathbf{J}(\mathbf{x})$

where $\mathrm{C}=\mathrm{CP}_{\max } \cap \mathrm{CQoS} \cap \mathrm{CP}_{\max } \mathrm{R}$ and each constraint in a linear form is as follow

$$
\begin{aligned}
& \mathcal{C}_{p_{\max }}: \frac{x_{i}}{g_{i}}-p_{\max } \leq 0, \forall i \in\{1,2, \cdots, M\} . \\
& \mathcal{C}_{Q o S}:-x_{i}+\alpha \Lambda \sum_{\substack{k=1 \\
k \neq i}}^{M} x_{k}+\Lambda I \leq 0, \forall i \in\{1,2, \cdots, M\} . \\
& \mathcal{C}_{P_{R}^{\max }}: \sum_{j=1}^{M} x_{j}-P_{R}^{\max } \leq 0 .
\end{aligned}
$$


With $2 M+1$ Lagrange multipliers $\left(\theta_{1}, \cdots, \theta_{M}, \lambda_{1}, \cdots, \lambda_{M}\right.$, $\mu$ ), the complete Lagrange, denoted as $\mathcal{L}$, becomes as follow:

$$
\begin{aligned}
\mathcal{L}= & J-\sum_{i=1}^{M} \theta_{i}\left(\frac{x_{i}}{g_{i}}-p_{\max }\right) \\
& -\sum_{i=1}^{M} \lambda_{i}\left(-x_{i}+\alpha \Lambda \sum_{k=1, k \neq i}^{M} x_{k}+\Lambda I\right) \\
& -\mu\left(\sum_{j=1}^{M} x_{j}-P_{R}^{\max }\right) \\
= & J-\sum_{i=1}^{M}\left(\theta_{i}+\mu-\lambda_{i}+\alpha \Lambda \sum_{k=1, k \neq i}^{M} \lambda_{k}\right) x_{i} \\
& + \text { some constant. }
\end{aligned}
$$

From the condition, for optimum $\mathbf{x}$ 's, we have $\partial \mathrm{L} / \partial \mathrm{xi}=0, \mathrm{i}=1,2, \cdot \cdot$, $\mathrm{M}$, so

$$
\frac{\partial J}{\partial x_{i}}=\frac{\theta_{i}}{g_{i}}+\mu-\lambda_{i}+\alpha \Lambda \sum_{k=1, k \neq i}^{M} \lambda_{k} .
$$

Also we have the "non-negativity" and the "complimentary slackness"; all 2M +1 Lagrange multipliers are nonnegative and if a constraint is met with strict inequality (non-binding),

then the corresponding method for finding the maximum and minimum of function with multiple variables that are under restriction multiplier is zero. Note that $\mathrm{CP}_{\max } \mathrm{R}$

is on all the users, so the value of $\mu$ is common to all the users. We can classify all $\mathrm{M}$ users into the following three disjoint groups according to the binding constraints - depending upon $\lambda \mathrm{i}$ and or $\theta \mathrm{i}$ are zero:

- Users with QoS -binding: Denote this group as A1. Then for user $\mathrm{j}, \mathrm{j} / \in \mathrm{A} 1, \lambda \mathrm{j}=0$, and for user $\mathrm{i}, \mathrm{i} \in \mathrm{A} 1$

$$
\frac{\partial J}{\partial x_{i}}=\frac{\theta_{i}}{g_{i}}+\mu-\lambda_{i}+\alpha \Lambda \sum_{k \neq i, k \in A_{1}} \lambda_{k} .
$$

We include $\theta \mathrm{i}$ in (12) in order to unify the possibility that a user may transmit at $\mathrm{P}_{\max }$ requirement, and $\theta \mathrm{i}=0$ is

if and only if user $\mathrm{i}$ transmits with strictly less than $\mathrm{P}_{\max }$

- Users with neither QoS nor peak-power binding: Denote this group as A2. Then, for user i, i $\in$ A2

$$
\frac{\partial J}{\partial x_{i}}=\mu+\alpha \Lambda \sum_{k \in A_{1}} \lambda_{k} .
$$

- Users with peak-power-binding but no QoS-binding: Denote this group as A3. Then, for user i, i $\in$ A3

$$
\frac{\partial J}{\partial x_{i}}=\frac{\theta_{i}}{g_{i}}+\mu+\alpha \Lambda \sum_{k \in A_{1}} \lambda_{k} .
$$

of the optimum solution _ conditioning that a polarities of $(\partial \mathrm{J} / \partial \mathrm{xi}-\partial \mathrm{J} / \partial \mathrm{xj})$ and $(\mathrm{xi}-\mathrm{xj})$ are either always the same or always the opposite. Define sign (.) as follow.

$$
\operatorname{sign}(x)= \begin{cases}\frac{x}{|x|}, & \text { for } x \neq 0, \\ 0, & \text { for } x=0 .\end{cases}
$$


One word on notation: in the following derivation, the term "QoS -achieving power" means the minimum power pi such that user i satisfies the QoS constraint when $\mathbf{p}-\mathrm{i}$ is fixed. This power level is not a constant, but dependent on $\mathbf{p}-\mathrm{i}$. Also "intermediate power" means strictly higher than the "QoS achieving power" but strictly less than the The maximum power, $\mathrm{P}_{\max }$.

Theory 1 For an optimum solution, there can be at most one user with intermediate power. In other words, number of users in group $\mathrm{A} 2$ is at most one.

Proof: Suppose that $x_{*}^{*}$ is a optimum solution of (6), with $x^{*} i=p^{*} g$ i gi and $x^{* j}$ between their respective QoSachieving powers and $\mathrm{P}_{\max }$. As user $\mathrm{i}$ and $\mathrm{j}$ are both in group A2, from (13) we have $\partial \mathrm{J} / \partial \mathrm{xi}=\mathrm{Sign}(\partial \mathrm{J} / \partial \mathrm{xi}-$ $\partial \mathrm{J} / \partial \mathrm{xj})=\operatorname{sign}(\mathrm{xi}-\mathrm{xj})$

$$
\mathrm{x}_{\delta}^{*}=\left(x_{1}, \cdots, x_{i}+\delta, \cdots, x_{j}-\delta, \cdots, x_{M}\right) \text {, with } \mathrm{x}_{\delta}^{*} \in \mathcal{C} .
$$

Then,

$$
J\left(\mathrm{x}_{\delta}^{*}\right)-J\left(\mathrm{x}^{*}\right)=\int_{0}^{\delta} \frac{\partial J}{\partial \tilde{\delta}} d \tilde{\delta}
$$

where

$$
\frac{\partial J}{\partial \tilde{\delta}}=\left.\left(\frac{\partial J}{\partial x_{i}}-\frac{\partial J}{\partial x_{j}}\right)\right|_{\mathbf{x}=\mathbf{x}_{\tilde{\delta}}^{*}}>0, \quad \text { for any } 0<\tilde{\delta}<\delta \text {. (17) }
$$

Therefore, we can find $\mathrm{x}_{\delta}^{*} \in \mathcal{C}$, such that $J\left(\mathrm{x}_{\delta}^{*}\right)>J\left(\mathrm{x}^{*}\right)$.

Therefore, we can find $\mathbf{x}^{*} \delta \in \mathrm{C}$, such that $\mathrm{J}\left(\mathbf{x}^{*} \delta\right)>\mathrm{J}\left(\mathbf{x}^{*}\right)$. This is a opposition. In the following, we present an approach to find the optimum solution of (6) under the same-sign situation by decreasing the solution space. Reduction of the solution space can be done by ignoring some forms of $\mathbf{x}$ without losing optimality. A specific form of $\mathbf{x}$ is ignored as a potential optimum solution, if an optimum solution cannot exist with that specific form, or we can find $\mathbf{y}$ in another form of consideration such that $\mathrm{J}(\mathbf{y})=\mathrm{J}(\mathbf{x})$. Theory 2 characterizes the solution space when there is no intermediate user. When there exists one and only one intermediate user, Theory 3 and 4 delineates the distribution of users in group A1 and A3.

Theory 2 Considering the case when there is no intermediate user, we can restrict our attention of an optimum solution to the following cases without losing optimality; any user with $\mathrm{P}_{\max }$ has a higher channel gain than the users with QoS-achieving.

Proof: Suppose that $\mathbf{x}^{*}$ is an optimum solution of (6).

with $\mathrm{x}^{*} \mathrm{k}=\mathrm{P}_{\max } \mathrm{g}_{\mathrm{k}} \quad$ or $\gamma^{*} \mathrm{k}=\Lambda$, for all $\mathrm{k} \in\{1,2, \cdots, \mathrm{M}\}$, i.e. no intermediate user. Suppose that gi $>\mathrm{gj}$, pi $<\mathrm{P}_{\max }$ and $\gamma \mathrm{j}>\Lambda$, i.e. user $\mathrm{i}$ and $\mathrm{j}$ belong to group $\mathrm{A} 1$ and $\mathrm{A} 3$,

respectively. From (13) and (14), $(\partial \mathrm{J} / \partial \mathrm{xi}<\partial \mathrm{J} / \partial \mathrm{xj})$ and from the same-sign condition, $\mathrm{x} * \mathrm{i}<\mathrm{x} * \mathrm{j}$. Define $\mathbf{x}^{* *}$ such that $\mathrm{x} * \mathrm{k}=\mathrm{x} * \mathrm{k}$ for $\mathrm{k} \in\{1,2, \cdots, \mathrm{M}\}$, except $\mathrm{x} * \mathrm{I}=\mathrm{x} *$

$\mathrm{j}$ and $\mathrm{x}^{* * \mathrm{j}}=\mathrm{x} * \mathrm{i}$. As $\mathrm{x}^{* \mathrm{i}}<\mathrm{x} * \mathrm{j}, \mathbf{x}^{* *} \in \mathrm{C}$. Note that $\mathrm{J}\left(\mathbf{x}^{*}\right)=\mathrm{J}\left(\mathbf{x}^{* *}\right)$ while $\mathbf{x}^{* *} \in \mathrm{C}$, and $\mathbf{x}^{* *}$ has one intermediate user of user $\mathrm{j}$. Therefore, if $\mathbf{x}^{*}$ is the optimum solution without an intermediate user, then we can find $\mathbf{x}^{* *}$ which is also an optimum solution with one intermediate user.

Theory 3 If an optimum solution has one and only one intermediate user, the users with lower channel gains than the intermediate user's, meet the QoS-constraint with equality.

Proof: Suppose that $\mathrm{x}^{*}$ is an optimum solution of (6) and there exists $\mathrm{i}$ and $\mathrm{j}$ such a gi $>$ gj $\mathrm{p}^{* \mathrm{i}}\left\langle\mathrm{P}_{\max }, \gamma^{*} \mathrm{i}>\Lambda\right.$ and $\gamma^{*} \mathrm{j}>\Lambda$. Following the same way as in the proof of Lemma

2 , by exchanging the received power of user $\mathrm{i}$ and $\mathrm{j}$, we can find $\mathbf{x}^{* *} \in \mathrm{C}$ such that $\mathrm{J}\left(\mathbf{x}^{*}\right)=\mathrm{J}\left(\mathbf{x}^{* *}\right)$. Then, $\mathbf{x}^{* *}$ has two

intermediate users - user $\mathrm{i}$ and $\mathrm{j}$. From Lemma 1, we can find $\mathbf{x}^{* * * \in C} \mathrm{C}$ such that $\mathrm{J}\left(\mathbf{x}^{* * *}\right)>\mathrm{J}\left(\mathbf{x}^{* *}\right)=\mathrm{J}(\mathbf{x})$. This is a contradiction. 
Theory 4 If an optimum solution has one and only one intermediate user, without losing optimality, we can restrict our attention of the optimal solution such that all users with higher channel gains than the intermediate user's, transmit with $\mathrm{P}_{\max }$.

Proof: Suppose that $\mathbf{x}^{*}$ is an optimum solution of (6) and there exists $\mathrm{i}$ and $\mathrm{j}$ such that gi $>$ gj, $\mathrm{p}^{* \mathrm{i}}$ $<\mathrm{P}_{\max }, \gamma^{* \mathrm{i}}=\Lambda$ and $\mathrm{P} * \mathrm{j}<, \mathrm{P}_{\max } \gamma^{* \mathrm{j}}>\Lambda$, i.e. user $\mathrm{i}$ is a QoS- binding user and user $\mathrm{j}$ is an intermediate user. Then, by swapping xi and $\mathrm{xj}$, we can achieve the same objective under the same constraint. By doing so, without losing optimality, we can ignore optimum solutions such that the intermediate user (the user in groupA2), if there is, has lower channel gain than the users with QoS-binding (the users in group A3). _ From Theory 1 to 4 , an optimal solution can be found as follows. Slice the users into two classes - good and bad, based on their channel gains. For each of the $\mathrm{M}+1$ possible ways of segment users, allocate the transmit power in the following fashion. Allocate $\mathrm{P}_{\max }$ to all good users while the same received power $\mathrm{u}$ is received to all bad users just to meet the QoS requirement. There can be one exception -the user with the best channel gain among the bad users is allocated $(\mathrm{v}>\mathrm{u})$, and this user is the possible mediator user. Improve efficiency the purpose with respect to the two variables $u$ and $v$, subject to limitation. Appraise the purpose, and compare the objective values for each possible slicing rule, $u$, and v. Pick the slice, $u$ and $v$ that yields the best value. A summary, sign $(\partial \mathrm{J} / \partial \mathrm{xi}-\partial \mathrm{J} / \partial \mathrm{xj})_{-}=\operatorname{sign}(\mathrm{xi}-\mathrm{xj})$ implies a greedy policy, namely the good users transmit $\mathrm{P}_{\max }$, and bad users transmit its QoS -achieving power while there can be at most one user in-between. Application of this optimal policy is shown in Proposition 1 of Section IV-A.

C. Opposite-Sign Condition, i.e. $\operatorname{sign}(\partial \mathrm{J} / \partial \mathrm{xi}-\partial \mathrm{J} / \partial \mathrm{xj})=-\operatorname{sign}(\mathrm{xi}-\mathrm{xj})$

Like in Section III-B, the derivation starts with paying attention to the intermediate users; users belong to group A2.

Theory 5 For an optimum solution, if there are users with intermediate powers, their received powers should be the same.

Proof: Suppose that $\mathbf{x}^{*}$ is a optimum solution of (6) with

$\gamma^{*} \mathrm{i}>\Lambda, \mathrm{p}^{*} \mathrm{i}<\mathrm{p} \max$, and $\gamma^{* \mathrm{j}}>\Lambda, \mathrm{p}^{*} \mathrm{j}<\mathrm{p} \max$, i.e. user $\mathrm{i}$ and $\mathrm{j}$ are users with intermediate transmit power. Then both user $\mathrm{i}$ and $\mathrm{j}$ belong to group A2. from (13)

,$(\partial \mathrm{J} / \partial \mathrm{xi}=\partial \mathrm{J} / \partial \mathrm{xj})$ and

therefore from $\operatorname{sign}(\partial \mathrm{J} / \partial \mathrm{xi}=\partial \mathrm{J} / \partial \mathrm{xj})$

$=-\operatorname{sign}(x i-x j), x * i=x * j$.

The following Lemmas delineate the distributions of users in group A1 and A3, with respect to the users in A2.

Theory 6 Except the case where all the received powers are the same, if there exists a QoS-binding user, then the Qo $S$ binding user transmit with $\mathrm{P}_{\max }$.

Proof: Suppose that $\mathbf{x}^{*}$ is an optimum solution of (6) with

$\gamma^{*} \mathrm{i}=\Lambda, \mathrm{p}^{* \mathrm{i}}<\mathrm{P}_{\max }$, and $\gamma^{* \mathrm{j}}>\Lambda$, where $\mathrm{i}, \mathrm{j} \in\{1,2, \cdots, \mathrm{M}\}-$ in other words, user $\mathrm{i}$ belongs to A1 and user $\mathrm{j}$ belongs to $\mathrm{A} 2$ or A3. From (10) and (11), as $\theta \mathrm{i}=0$ due to $\mathrm{p} * \mathrm{i}<\mathrm{p} \max$,

$$
\begin{aligned}
& \frac{\partial J_{1}}{\partial x_{i}}-\frac{\partial J_{1}}{\partial x_{j}}=\left(I+\alpha \sum_{k=1}^{M} x_{k}\right) \\
& \quad \times\left\{\frac{1}{\left(I+\alpha \sum_{k=1, k \neq i}^{M} x_{k}\right)^{2}}-\frac{1}{\left(I+\alpha \sum_{k=1, k \neq j}^{M} x_{k}\right)^{2}}\right\} .
\end{aligned}
$$

Then we have

$$
\begin{aligned}
\operatorname{sign}\left(\frac{\partial J_{1}}{\partial x_{i}}-\frac{\partial J_{1}}{\partial x_{j}}\right) & =\operatorname{sign}\left\{\frac{1}{\left(I_{0}+\alpha x_{j}\right)^{2}}-\frac{1}{\left(I_{0}+\alpha x_{i}\right)^{2}}\right\} \\
& =\operatorname{sign}\left(x_{i}-x_{j}\right) .
\end{aligned}
$$


Therefore, the optimum policy is greedy. Also, for (P2) from Section II-B, we have

$$
\begin{aligned}
\frac{\partial J_{2}}{\partial x_{i}} & =\frac{1}{I+x_{i}+\alpha \sum_{k=1, k \neq i}^{M} x_{k}} \\
& -\sum_{\substack{m=1 \\
m \neq i}}^{M} \frac{\alpha x_{m}}{\left(I+\alpha \sum_{\substack{k=1 \\
k \neq m}}^{M} x_{k}\right)\left(I+x_{m}+\alpha \sum_{\substack{k=1 \\
k \neq m}}^{M} x_{k}\right)} .
\end{aligned}
$$

After some manipulation, we have

$$
\begin{aligned}
& \operatorname{sign}\left(\frac{\partial J_{2}}{\partial x_{i}}-\frac{\partial J_{2}}{\partial x_{j}}\right) \\
& =\operatorname{sign}\left\{\begin{array}{l}
\left(I_{0}+\alpha x_{i}\right)\left(I_{0}+x_{j}+\alpha x_{i}\right) \\
-\left(I_{0}+\alpha x_{j}\right)\left(I_{0}+x_{i}+\alpha x_{j}\right)
\end{array}\right\} \\
& =\operatorname{sign}\left\{\left(x_{i}-x_{j}\right)\left(\alpha^{2}\left(x_{i}+x_{j}\right)+(2 \alpha-1) I_{0}\right)\right\} .
\end{aligned}
$$

If $\alpha \geq 1 / 2$, we $\operatorname{sign}-(\partial J 2 / \partial x \mathbf{x}-\partial J 2 / \partial x j)_{-}=\operatorname{sign}(x i-x j)$ and therefore a greedy policy is optimal. Since $\alpha \approx 1$ in practical systems [2], we have not lost too much generality.

Proposition (Optimality of the greedy standpoint) For (P1) and (P2), (if $\alpha \geq 12$ for (P2)), without losing optimality, we can restrict our attention of an optimal power allocation vector to the following form:

$$
\mathrm{p}^{*}=\left(p_{\max }, \cdots, p_{\max }, p_{i_{T}}^{*}, p_{i_{T}+1}^{*}, p_{i_{T}+2}^{*}, \cdots, p_{M}^{*}\right)
$$

where $i_{T}$ is an integer, $1 \leq i_{T} \leq M, 0<p_{i}^{*} \leq p_{\max }$ and

$$
\frac{p_{m}^{*} g_{m}}{I+\sum_{j=1}^{i_{T}-1} p_{\max } g_{j}+\sum_{j=i_{T}, j \neq m}^{M} p_{j}^{*} g_{j}}=\Lambda, \forall m>i_{T} .
$$

Proof: It can be readily shown from the same-sign property of J1 and J2.

B. Product Form of Optimization - (P3) and (P4) For (P3) from Section II-C, we have

$$
\begin{aligned}
\frac{\partial J_{3}}{\partial x_{i}} & =\frac{1}{I+\alpha \sum_{k=1, k \neq i}^{M} x_{k}} \prod_{\substack{n=1 \\
n \neq i}}^{M} \frac{x_{n}}{I+\alpha \sum_{k=1, k \neq n}^{M} x_{k}} \\
& -\sum_{\substack{m=1 \\
m \neq i}}^{M} \frac{\alpha x_{m}}{\left(I+\alpha \sum_{k=1, k \neq m}^{M} x_{k}\right)^{2}} \prod_{\substack{n=1 \\
n \neq m}}^{M} \frac{x_{n}}{I+\alpha \sum_{k=1, k \neq n}^{M} x_{k}} \\
& =J_{3} \times\left\{\frac{1}{x_{i}}-\sum_{\substack{m=1 \\
m \neq i}}^{M} \frac{\alpha}{\left(I+\alpha \sum_{k=1, k \neq m}^{M} x_{k}\right)}\right\}
\end{aligned}
$$

After deleting the common terms, we have the following simple relationship 


$$
\begin{aligned}
\operatorname{sign}\left(\frac{\partial J_{3}}{\partial x_{i}}-\frac{\partial J_{3}}{\partial x_{j}}\right) & =\operatorname{sign}\left\{\frac{1}{x_{i}\left(I_{0}+\alpha x_{i}\right)}-\frac{1}{x_{j}\left(I_{0}+\alpha x_{j}\right)}\right\} \\
& =-\operatorname{sign}\left(x_{i}-x_{j}\right)
\end{aligned}
$$

It implies that a fair standpoint is optimum. Also, for (P4) from Section II-D, we establish the relationship between $\operatorname{sign}(\partial \mathrm{J} 4 / \partial \mathrm{xi}-\partial \mathrm{J} 4 / \partial \mathrm{xj})$ and $\operatorname{sign}(\mathrm{xi}-\mathrm{xj})$ as follow.

For convenience, define

$$
L_{i}=\log \left(1+\frac{x_{i}}{I+\alpha \sum_{k=1, k \neq i}^{M} x_{k}}\right) .
$$

For convenience, define

The partial derivative is shown in (27). After canceling common terms, we have

$$
\begin{aligned}
& \frac{\partial J_{4}}{\partial x_{i}}-\frac{\partial J_{4}}{\partial x_{j}} \\
& =J_{4} \times\left\{\begin{array}{l}
\frac{\left(I_{0}+\alpha x_{i}+\alpha x_{j}\right)}{L_{i}\left(I_{0}+\alpha x_{j}\right)\left(I_{0}+x_{i}+\alpha x_{j}\right)} \\
-\frac{\left(I_{0}+\alpha x_{i}+\alpha x_{j}\right)}{L_{j}\left(I_{0}+\alpha x_{i}\right)\left(I_{0}+x_{j}+\alpha x_{i}\right)}
\end{array}\right\} .
\end{aligned}
$$

Define

$$
\Delta=L_{i}\left(I_{0}+\alpha x_{j}\right)\left(I_{0}+x_{i}+\alpha x_{j}\right)-L_{j}\left(I_{0}+\alpha x_{i}\right)\left(I_{0}+x_{j}+\alpha x_{i}\right),
$$

then we have

$$
\operatorname{sign}\left(\frac{\partial J_{4}}{\partial x_{i}}-\frac{\partial J_{4}}{\partial x_{j}}\right)=-\operatorname{sign}(\Delta) .
$$

Next, we connect sign $(\Delta)$ with sign $(x i-x j)$. Let $x j$ be fixed and the domain of $x i$ is $[0, \infty]$.

At $\mathrm{xi}=0, \Delta<$ 0 , and as $\mathrm{xi} \rightarrow \infty, \Delta \rightarrow \infty$. So a root for the equation $\Delta(\mathrm{xi})=0$, must exist. In the following, it is shown that $\mathrm{xi}=$ $\mathrm{xj}$ is the unique root. As a reference, we have

$$
\begin{aligned}
& \frac{\partial L_{i}}{\partial x_{i}}=\frac{1}{I_{0}+x_{i}+\alpha x_{j}}, \text { and } \\
& \frac{\partial L_{j}}{\partial x_{i}}=-\frac{\alpha x_{j}}{\left(I_{0}+\alpha x_{i}\right)\left(I_{0}+x_{j}+\alpha x_{i}\right)} .
\end{aligned}
$$

Then,

$$
\begin{aligned}
\frac{\partial \Delta}{\partial x_{i}}= & \left(I_{0}+2 \alpha x_{j}\right)+\log \left(1+\frac{x_{i}}{I_{0}+\alpha x_{j}}\right)\left(I_{0}+\alpha x_{j}\right) \\
& -\alpha \log \left(1+\frac{x_{j}}{I_{0}+\alpha x_{i}}\right)\left(2 I_{0}+2 \alpha x_{i}+x_{j}\right) .
\end{aligned}
$$




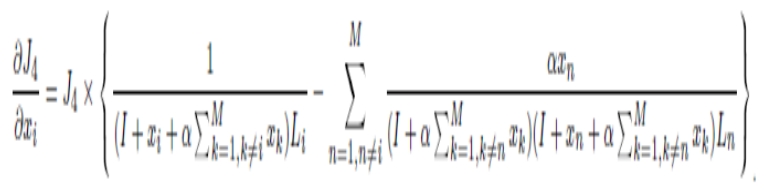

At $x i=0$, the sign of (33) is not clear; but as $x i \rightarrow \infty,(\partial \Delta / \partial x i) \rightarrow \infty$. However, this is not enough to determine the sign of $\Delta \operatorname{or}(\partial \Delta / \partial \mathrm{xi})$ with respect to the sign of $(\mathrm{xi}-\mathrm{xj})$. So taking derivative again:

$$
\begin{aligned}
\frac{\partial^{2} \Delta}{\partial x_{i}{ }^{2}}= & \frac{I_{0}+\alpha x_{j}}{I_{0}+x_{i}+\alpha x_{j}}+\frac{\alpha^{2} x_{j}\left(2 I_{0}+2 \alpha x_{i}+x_{j}\right)}{\left(I_{0}+\alpha x_{i}\right)\left(I_{0}+\alpha x_{i}+x_{j}\right)} \\
& -2 \alpha^{2} \log \left(1+\frac{x_{j}}{I_{0}+\alpha x_{i}}\right) .
\end{aligned}
$$

Again, at $x i=0$, the sign of (34) is not clear; but when $x i \rightarrow \infty$, we have $\partial 2 \Delta / \partial x i$ In order to determine the sign of , the third derivative can be investigated. After some algebraic manipulation, we have $\frac{\partial^{2} \Delta}{\partial x_{i}{ }^{2}} \sim \frac{1}{x_{i}}>0$.

$$
\begin{aligned}
\frac{\partial^{3} \Delta}{\partial x_{i}{ }^{3}}= & -\frac{I_{0}+\alpha x_{j}}{\left(I_{0}+x_{j}+\alpha x_{j}\right)^{2}} \\
& -\frac{\alpha^{3} x_{j}^{3}}{\left(I_{0}+\alpha x_{i}\right)^{2}\left(I_{0}+\alpha x_{i}+x_{j}\right)^{2}}<0 .
\end{aligned}
$$

Now finally can conclude that $\left(\partial_{2} \Delta / \partial_{2} \mathrm{xi}\right)$ a decreasing function of xi. As $\left(\partial_{2} \Delta / \partial_{2} \mathrm{xi}\right)$ is positive at $\mathrm{xi} \rightarrow \infty$, it is positive for all $\mathrm{xi}>0$. The fact that $\left(\partial_{2} \Delta / \partial_{2} \mathrm{xi}\right)>0$,implies that $(\partial \Delta / \partial \mathrm{xi})$ is a monotonically increasing function of xi. There are two cases to consider

- Case $1:(\partial \Delta / \partial x i)>0$ for all $x i>0$;

- Case $2:(\partial \Delta / \partial \mathrm{xi})<0$ for small xi but $(\partial \Delta / \partial \mathrm{xi})>0$ for large xi. Since $\Delta<0$ at xi=0 but $\Delta>0$ when xi large, in either case, the root of $\Delta(x i)=0$ is unique. The unique root is $x i=x j$, and the uniqueness of the root implies $\operatorname{sign}(\Delta)=\operatorname{sign}(x i-x j)$. Overall, from (30) we have the conclusion that

$$
\operatorname{sign}\left(\frac{\partial J_{4}}{\partial x_{i}}-\frac{\partial J_{4}}{\partial x_{j}}\right)=-\operatorname{sign}\left(x_{i}-x_{j}\right) .
$$

So (P4) falls into the category that a fair policy is optimum.

Proposition 2 (Optimality of the fair policy) For (P3) and (P4), without losing optimality, we can restrict our attention of the optimal power allocation vector to the following form:

$\mathbf{P}^{*}=\left(\mathrm{p}^{* 1} 1, \mathrm{p}^{*} 2, \cdots, \mathrm{p} * \mathrm{iG}, \mathrm{P}_{\max }, \mathrm{P}_{\max }, \cdots, \mathrm{P}_{\max }\right)$

Where $\mathrm{iG}$ is an integer, $1 \leq \mathrm{iG} \leq \mathrm{M}$, and $\mathrm{p} * 1 \mathrm{~g} 1=\mathrm{p} * 2 \mathrm{~g} 2=\cdots=\mathrm{p} * \mathrm{iG}$ giG $\geq \mathrm{P}_{\max }$ giG +1

. Proof: It can be readily shown from the opposite-sign property of J3 and J4 


\title{
V. Numerical Examples
}

In this section, numerical examples for the total capacity under both sum throughput maximization and product throughput maximization are present. The impact of the QoS requirements is also discussed. Table I summarizes the formation used in the numerical examples. Channel models and their related parameters are from [20]. Users are randomly

\section{NUMERICAL RESULT}

\author{
Cell-Structure - Single Hexagonal cell \\ Cell Radius $-1.2 \mathrm{~km}$ \\ Antenna Radiation - Omni-directional \\ Maximum transmit power form mobile \\ station -0.2 watt
}

(without variation distributed) dropped over a single hexagonal cell, where the base station is located at the center of the cell. A set of dropped users is considered for the capacity computation only if there exists a power portion solution that guarantees the minimum QoS requirements are satisfied. Otherwise, the entire users are re-dropped. In practice, the probability condition is assured by network planning and admission control [19]. Each user is apportioned with a stationary channel gain, gi, taking into account distance loss and log-normal shadowing. Since the optimization problem does not take into account the time varying radio condition, fast fading and power control aspects are not considered in this study. The Rise-Over-Thermal (ROT) is a limitation on the total received power at the base station, and is defined in Table I. Fig. 1 represents the capacity obtained when the transmit powers of the users are allocated to maximize the sum capacity. All through this section, all the capacity numbers are normalized by the total bandwidth so that their unit is 'Bits/Sec/Hz'. In the "No-QoS requirement" case $(\Lambda=0)$, the multi-user diversity gain (practice of seizing opportunities scheduling gain) is observed with a lessening return. On the other hand, if there is a QoS requirement, the multi-user penalties (capacity decrease by increasing the number of users) are observed. As the number of the users increase, the statistics of the poor users gets worse, and more consequentially the statistics of the poor channel quality users can drag the system capacity. Basically, as the maximum sum-capacity power allocation (summation form) is a greedy policy, the QoS requirements (fairness constraint) impacts a lot on the capacity of the system. Note that QoS requirements $(-21 \mathrm{~dB}$ and $-18 \mathrm{~dB})$ are chosen to represent the typical SINR level for $9.6 \mathrm{Kbps}$ traffic channel throughput maintenance and the reverse pilot channel maintenance, respectively [21].Fig. 2 represents the capacity obtained when the powers are allocated to maximize the product of the user capacities.

In this policy, even without a QoS-constraint, the power allocation strategy leads to a fair standpoint, and therefore the multi-user penalties are observed. Unsuitability QoS constraints.

portion. We have also studied the impact of different level of QoS constraint on the sector/cell performance. Our future work includes the investigation of possible independent transmission scheme to achieve the optimal allotment. We also need to study multi-cell coordination and the extension to optimizes considering channel the variation

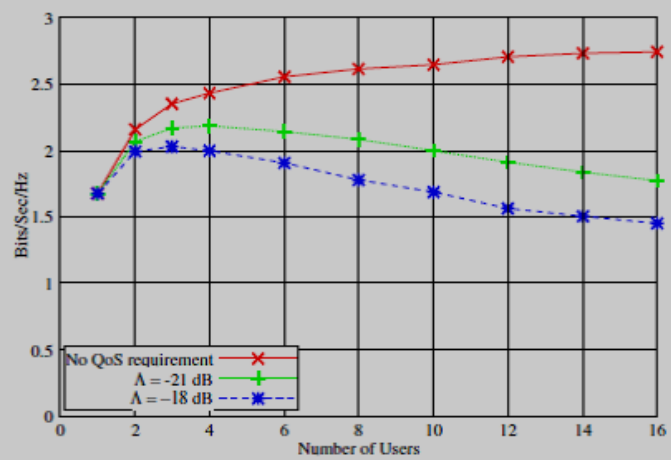

Fig. 1. Capacity of the system when the powers are allocated to maximize the sum-capacity of the system with different QoS requirements. 


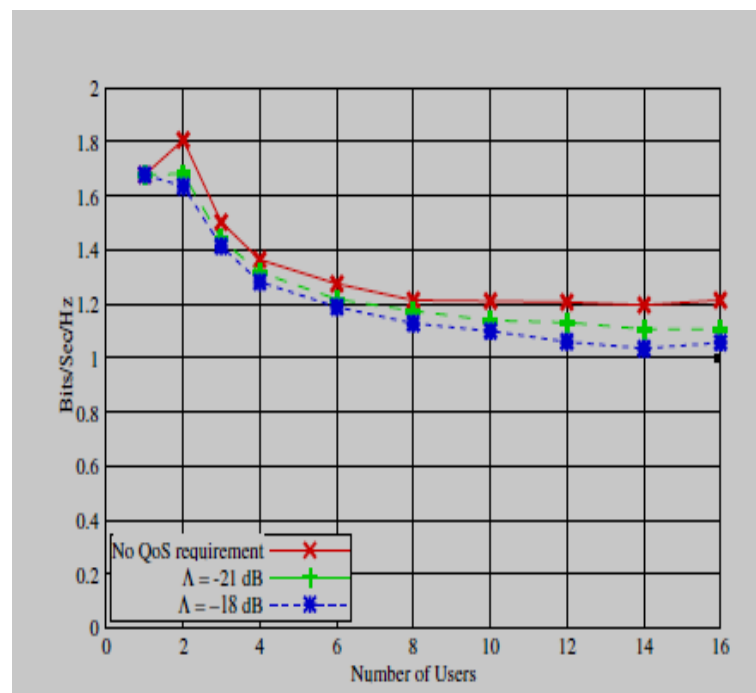

Fig. 2. Capacity of the system when the powers are allocated to maximize the product of user capacity with different QoS requirements.

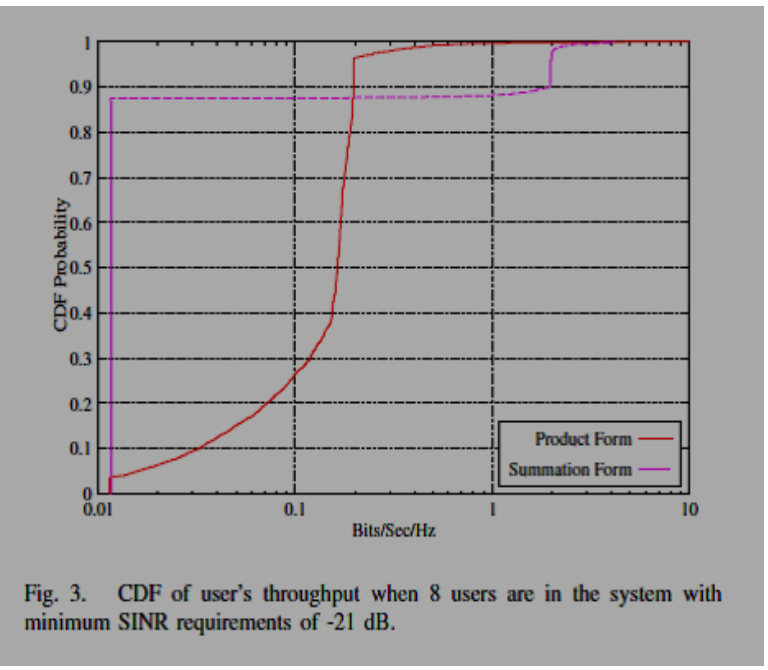

power, total received power and QoS constraints. Throughput maximization results in a greedy power allocation whereas the -throughput largest possible magnification results in a 'proportional fair' allocation. We have also studied the impact of different level of QoS constraint on the sector/cell performance.

Our future work includes the investigation of possible autonomous transmission scheme to achieve the optimal allocations.

We also need to study multi-cell coordination and the extension to optimizations considering channel variation.

\section{References}

[1]. Optimal resource allocation in multi-service CDMA Network.”IEEE Trans. Wirless communication.

[2]. J. walrand, "fair pir to pir window based congestion control, ,IEEE networking.

[3]. CDMA: principal of spread spectrum communication Addison- Wesley 1995.

[4]. Power control and capcity of spread wirless network 1987- Dec.1999.

[5]. Data throughput of CDMA - HDR a high data rate personal communication wirless system, may 2000,pp-1854-1858.

[6]. Wirless network Evolation 3G upper Saddle River, prentice hall 2002.

[7]. L.wang transfer control protocol A duality model June 2000.

[8]. Information capacity and power control in single-cell communication in IEEE, June 1995

[9]. Wirless commncations and Networking 2004,WCNC,2004 IEEE (volume-3)

[10]. Vehicular technology coference, 2006 VTC 2006,IEEE 63 (volume).

[11]. Mathmatical modeling of the Internet in mathmetics unlimited-2001 and Beyoned 2001,p.p 685-702.

[12]. E.Estenes "An algorithm for reverse traysic channel rate control for CDMA 2000 high rate packet data system.IEEE-2001.

[13]. Q.wu,'Link budget of CDMA $20001 \mathrm{xev}$ do wirless Internet access system in proc.13 ${ }^{\text {th }}$ IEEE mobile Radio communication2002,vol-4

[14]. Admission contol schemes for wirless communication networks with adjustable transmitter power IEEE INFOCOM 94. 
[15]. E.Estene, on the reverse link performance of CDMA 2000 high rate packet system ,in proc IEEE INTERNATIONALCOMMUNCATIONCONFERNCE -2002.

[16]. Note on effective bandwidth in Stochastics Networks. Theory and application.I.Ziedins. EDS.Oxford university press 1996.

[17]. $\quad 3^{\text {rd }}$ Generation partnership project $2(3 \mathrm{Gpp} 2) . c$ dma 2000 high rate packet data Interfernce Specification Technical report C.S 0024.A.V2.0 July 2005.

[18]. D.Bertsekas, J.Tesitskilis, parallel and Distributed computation.prentice-Hall Inc. 1989

[19]. T.Janidi "on dual method for adaptive resource allocation in wirless networks a taxonomy of practical challenges in CDMA in Resource Allocation in Next Generation wireless Networks ,y.pan and Nova science publishers.Inc.2006.

[20]. P. Black and Q.wu" Link Budget of CDMA-2000 1Xev-do wireless Internet access system." InProc.13 ${ }^{\text {th }}$ IEEE International symposium on personal, Indoor and mobile radio communication-2002, Vol.4.

[21]. R.Horn and C.Johnson, Matrix Analysis Cambridge university press 1985

[22]. S.H Low and D.E Lapsley," Optimization Flow control, I: basic algorithm and convergence. IEEE/ACM

[23]. Trans. Networking vol.7, no.6, Dec-1999.

[24]. B. Radunovic and J. LeBoudec, Rate performance objectives of multi-hop wireless network in proc .INFOCOM 04

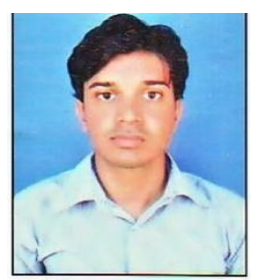

- $\quad$ AUTHOR INTRODUCTION:- Prof. Nitish Meena had been done his Graduation in B.TECH from Rajasthan techanical university, Kota (RTU).which is Affiliated from AICTE,New Delhi \& post Graduation in M.TECH from (S.U), Rajasthan, Affilited From AICTE\& U.G.C After that he is pursuing of Doctrate (Ph.D) Department of Eletronics \& Communcation. In Pratap University ,jaipur (Rajasthan). He have three year teaching Experience in his field \& recently Works as a vice-principal in SIET Engineering college, Lalsot (jaipur) And continuously researching in his field.

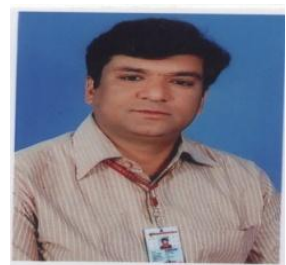

- $\quad$ AUTHOR INTRODUCTION:- Dr. Nilesh Parihar had been done his Graduation in B.TECH from Bangalor techanical university,(Tumkur).which is \& post Graduation in M.TECH from M.B.M Engineering college, J.N.V University, Jodhpur Rajasthan, Affilited From AICTE. After that he is Doctrate (Ph.D) Department of Eletronics \& Communcation. In Jodhpur National University, Jodhpur (Rajasthan). He have Fifteen year teaching Experience in his field \& recently Works as a principal in Bhartiya Institute of Engineering \& Technology, sikar (Rajasthan) And continuously researching in his field. 